\title{
Analytical Outage Probability for max-based Schedulers in Delay-Constrained Applications
}

\author{
Johannes Gonter, Norbert Goertz, Andreas Winkelbauer \\ Institute of Telecommunications \\ Vienna University of Technology \\ Email: johannes.gonter@nt.tuwien.ac.at
}

\begin{abstract}
The calculation of exact outage probabilities in delayconstrained multiuser-systems has been an unsolved problem. This paper introduces an analytical method to calculate the probability of an outage for max-based schedulers, which take a scheduling decision by choosing the user with an associated maximum metric. While this analysis is, therefore, suited for purely opportunistic scheduling, it is also suited for proportional fair scheduling, the arguably most important scheme in today's wireless systems. The approach presented in this paper does not restrict key system properties in any way: it can be applied to an arbitrary number of users with arbitrary channel statistics and arbitrary delay constraints. In order to prove the practicability, the outage probability is calculated for opportunistic and proportional fair scheduling scenarios, and it is shown to perfectly match the results of extensive numerical simulations. Furthermore, the most important practical implementation issues are discussed, and a new and highly useful interpretation of the maximum order statistic of i.n.i.d. (independent non-identically distributed) random variables is introduced.
\end{abstract}

Index Terms-Proportional Fair Scheduling, Outage Probability, Hard Delay Constraints, Block-Fading AWGN

\section{INTRODUCTION}

The ability of modern wireless communication systems to deliver some service with a certain reliability is often included in the notion of a "quality-of-service". This is, however, a very flexibly used term that may mean entirely different things in different situations. The term "outage", on the other hand, though very often seen as an aspect of QoS, describes the most crucial ability of a wireless multiuser system - the ability to deliver a certain amount of information within a given and usually limited period of time. This paper introduces an algorithm to precisely calculate the probability of such an outage for max-based scheduling schemes. This family of schedulers can be characterized by assigning a figure of merit $(F O M)$ to each user, selecting the user with the maximum FOM for scheduling - two prominent scheduling schemes based on this approach, and therefore within the scope of the present analysis, are opportunistic [1] and proportional fair scheduling [2].

While proportional fair scheduling has, due to its popularity and use in modern wireless networks, drawn a lot of attention, there seems to be a lack of analysis concerning its "shortterm" behavior with respect to hard ${ }^{1}$ delay constraints. While

\footnotetext{
${ }^{1}$ The definition of a hard delay constraint is detailed below.
}

proportional fairness has been investigated for fairness in delay-constrained environments [3], [4], most publications address cell throughput analyses [4], [5] rather than throughput for individual users. Also, asymptotic analyses [6], [7] have usually been performed rather than analyses that take care of practically important short-term availability of throughput. Furthermore, perhaps for mathematical tractability, many analyses specialize on Rayleigh fading assumptions [8], [7].

The algorithm presented in this paper calculates the outage probability for a specific set of parameters, which include hard delay constraints, arbitrary channel statistics for an arbitrary number of users, a constant transmit-power of every user, and a receiver noise-level for every user. The present analysis can even be applied to users with an arbitrary number of antennas (as long as selection diversity is applied).

The paper is organized as follows. Section II introduces the channel model. Section III contains an analytic solution to the problem at hand, which is specialized in section IV to opportunistic and proportional fair scheduling. Section V concludes the paper.

\section{Channel Model}

This paper elaborates on the downlink case with perfect Channel State Information (CSI) at the transmitting base-station serving $U$ users; CSI is required for the scheduler to take optimum scheduling decisions. The following analysis applies a block-fading AWGN channel model for which the "Gaussian Shannon-capacity" is assumed to be achieved within each block. However, the results of the analysis can be extended to non-ideal systems by assuming a power margin. An outage is defined as the event that the sum rate $R_{\text {sum }}$ (a random variable) transmitted within a window of $N$ consecutive blocks is below a required rate $r_{\text {sum }}^{\text {rea }}$ (a given number, typically determined by the service constraints of an application). This definition also clarifies the notion of a "hard" delay constraint: there is no possibility of carrying over "excess-information" transmitted within one window of $N$ consecutive blocks to the next window during which a required rate has to be achieved. This is a realistic assumption, e.g., in wireless multimedia transmission where a source signal is divided into blocks of samples (e.g. speech/audio samples or pixels) and each block is separately source-encoded and transmitted. At the receiving end, all source-bits that belong to a block of samples must be received "in time"; otherwise the source signal can not be 
reconstructed $^{2}$ rendering the block of received data useless. The analysis does not distinguish the cases where these $N$ block windows overlap or not: the probability of an outage is strictly defined as the probability that $R_{\text {sum }}<r_{\text {sum }}^{\text {req }}$ in any window of $N$ consecutive blocks.

For the statistical analysis of the outage probability to hold, uncorrelated channel-coefficients (both between users and individually for each user) are assumed. Without loss of generality, the probability of an outage is calculated for one distinct user $\left(u_{1}\right.$, representing user 1$)$, given the statistical channel knowledge of all users in the system.

\section{ANALYSIS OF max-BASED SCHEDULING}

As pointed out above, the fundamental approach presented in this paper does not distinguish different scheduling schemes, as long as the core-decision is memoryless and based solely on a max-operation to identify the user with the currently best FOM. This FOM may be the channel-power coefficients $|h|^{2}$ in case of opportunistic scheduling (which is fully equivalent to taking the rate $R$ achievable in the current block into account), or it may be the normalized current rate $\bar{R}=R / T$ in case of proportional-fair scheduling, with $T$ denoting the long-term average of rates achieved in the past. To preserve most general validity, the following analysis will therefore not deal with rates or channel-coefficients per se, but will consider FOMs exclusively, so that the same analysis can directly be applied to both opportunistic and proportionalfair scheduling. Specific results are subsequently presented in Section IV. Please note that the analysis presented in this paper is, in fact, not limited to strictly max-based scheduling. With the same approach, also scheduling schemes performing minbased scheduling (such as minimization of interference) can be assessed, since

$$
\arg \min \left\{x_{1}, x_{2}, \ldots x_{U}\right\}=\arg \max \left\{-x_{1},-x_{2}, \ldots-x_{U}\right\} .
$$

The presented considerations are therefore applicable to all scheduling schemes based on the search for extremums, however, all examples given in this paper refer to max-based schemes.

\section{A. Outage Probability of max-Based Scheduling}

Based on the above presented naming conventions, the probability of an outage for user 1 is the following sum of probabilities of mutually exclusive events:

$$
P_{1, \text { out }}\left(x_{1, \text { sum }}^{\text {req }}\right)=\sum_{k=0}^{N} P\left\{X_{1, \text { sum }}<x_{1, \text { sum }}^{\text {req }} \mid S_{1}=k\right\} P\left\{S_{1}=k\right\} .
$$

Here, $X_{1 \text {,sum }}$ denotes the sum of the FOMs in individual blocks of user $u_{1}$ that is achieved within a window of $N$ consecutive

\footnotetext{
${ }^{2}$ Although embedded source coding schemes (or multiple descriptions) may be used that produce a "basic" quality from a subset of the transmitted source bits, those techniques are not widely used, because embedded multimedia coding does often not produce a satisfactory quality trade-off compared with the quality-level of non-embedded coding at various rate-levels transmitted in parallel (multi-cast).
}

blocks, i.e. $N$ expresses a hard delay constraint. The quantity $x_{1 \text {,sum }}^{\text {req }}$ is the sum of FOMs that is requested ${ }^{3}$ to be achieved within a window of $N$ blocks; if it is not achieved an outage event occurred, and the goal in what follows is to analytically compute this outage probability. $S_{1}$ denotes the number of times user 1 is scheduled out of $N$ possible times within a window of length $N$ blocks: $S_{1}$ is, therefore, an integer-valued random variable ranging from 0 to $N$. Throughout the paper, the algorithms are developed to compute the probability of an outage for user 1 . If the probability of an outage shall be calculated for a different user, this can be achieved by reordering of the user indices; hence, no generality is lost.

The first summand of (2) (for $k=0$ ) can be simplified to $P\left\{S_{1}=0\right\}$, since an outage will occur when the user is not scheduled at all, i.e.,

$$
P\left\{X_{1, \text { sum }}<x_{1, \text { sum }}^{\text {req }} \mid S_{1}=0\right\}=1 .
$$

Analysis of (2) reveals two different structures: the expressions $P\left\{X_{1, \text { sum }}<x_{1 \text {,sum }}^{\text {req }} \mid S_{1}=k\right\}$ on the one hand, and the probabilities, $P\left\{S_{1}=k\right\}$, for user 1 to be scheduled $k$ times on the other hand. Following this structure, the mathematical derivation of the outage-calculation for $\max$-based schedulers is organized accordingly: In Section III-B, the expression $P\left\{S_{1}=k\right\}$ is analyzed, and in Section III-C the expressions of the form $P\left\{X_{1 \text {,sum }}<x_{1 \text {,sum }}^{\text {req }} \mid S_{1}=k\right\}$ are explored.

\section{B. Probability of User 1 being Scheduled $k$ Times.}

Given that user 1 can only be scheduled once or twice or three times, etc (where "or" is exclusive) the event "user 1 is scheduled a times within $N$ blocks" is distinct from the event "user 1 is scheduled $b$ times within $N$ blocks" for $a \neq$ $b$. Therefore, $P\left\{S_{1}=k\right\}$ can be calculated by drawing a probability tree of depth $N$, with $p_{1}$ being the probability that user 1 is scheduled in a block, and $1-p_{1}$ meaning the opposite. Therefore,

$$
P\left\{S_{1}=k\right\}=\left(\begin{array}{c}
N \\
k
\end{array}\right) \cdot p_{1}^{k} \cdot\left(1-p_{1}\right)^{(N-k)} .
$$

The actual calculation of an outage probability requires $p_{1}$ to be known, with $p_{1}$ being the probability that user 1 is scheduled in a particular block. From a max-based scheduling perspective this is equivalent to the current FOM $X_{1}$ of user 1 being larger than the current FOMs $X_{2}, \ldots, X_{U}$ of all other users $u_{2}, \ldots, u_{U}$. These random variables (or their realizations) could be rates in the case of opportunistic scheduling or normalized rates in case of proportional-fair scheduling or something entirely different. It is only important for this analysis that these FOMs are compared, and the user associated with the maximum FOM is chosen to be scheduled in the current block (out of $N$ blocks, over which the sum of FOMs is calculated to determine the outage probability by (2)). The "current" FOMs are going to be called $X_{u}$, with $X_{1}$ the $F O M$ of user 1 (the user of interest). Since all competing users (i.e. the users associated with FOMs $X_{2}, \ldots, X_{U}$ ) are

\footnotetext{
${ }^{3} x_{1, \text { sum }}^{\text {req }}$ is a number such as a bit rate requested by an application
} 
compared to user 1 in a memoryless max-operation, the first step is to simplify the problem by only considering the largest FOM among all competing users, and this FOM will be denoted by $Y$. This approach does not ignore any information available, since the statistics of all users are implicitly taken into account. With those assumptions, we state the following theorem:

Theorem 1. The probability $p_{1}$ that the FOM $X_{1}$ of user 1 $\left(u_{1}\right)$ is larger than the FOMs $X_{2}, X_{3}, \ldots, X_{U}$ of all competing users $u_{2} \ldots u_{U}$ is given by

$p_{1} \doteq P\left\{X_{1}>Y\right\}=1-\sum_{u=2}^{U} \int_{-\infty}^{\infty}\left(\frac{d}{d y} F_{X_{u}}(y)\right) \prod_{\substack{k=1 \\ k \neq u}}^{U} F_{X_{k}}(y) d y$

$$
=\int_{-\infty}^{\infty}\left(\frac{d}{d y} F_{X_{1}}(y)\right) \prod_{k=2}^{U} F_{X_{k}}(y) d y
$$

where $Y \doteq \max \left\{X_{2}, X_{3}, \ldots, X_{U}\right\}$ and the cumulated density functions (cdfs) of the FOMs of the users are defined by $F_{X_{u}}(y) \doteq P\left\{X_{u}<y\right\}$.

Proof: (Theorem 1)

$$
\begin{aligned}
P\left\{X_{1}>Y\right\} & =\int_{-\infty}^{\infty} P\left\{X_{1}>Y \mid Y=y\right\} f_{Y}(y) d y \\
& =\int_{-\infty}^{\infty}\left(1-F_{X_{1}}(y)\right) f_{Y}(y) d y \\
& =1-\int_{-\infty}^{\infty} F_{X_{1}}(y) f_{Y}(y) d y
\end{aligned}
$$

This leaves $F_{Y}(y)$, the cdf of the largest competing FOM, to be defined. As $F_{Y}(y)$ is the probability that all other users at the same time have a FOM smaller than $y$, statistical independence of all FOMs leads to the simple "product probability"

$$
F_{Y}(y)=P\{Y \leq y\}=\prod_{u=2}^{U} P\left\{X_{u} \leq y\right\}=\prod_{u=2}^{U} F_{X_{u}}(y)
$$

and the corresponding pdf $f_{Y}(y)$ is, therefore,

$$
\begin{aligned}
f_{Y}(y) & =\frac{d}{d y} F_{Y}(y)=\frac{d}{d y} \prod_{u=2}^{U} F_{X_{u}}(y) \\
& =\sum_{u=2}^{U}\left[\left(\frac{d}{d y} F_{X_{u}}(y)\right) \prod_{\substack{k=2 \\
k \neq u}}^{U} F_{X_{k}}(y)\right] .
\end{aligned}
$$

Hence, the probability of the FOM of user 1 being larger than those of the competing users can be expressed as:

$$
\begin{aligned}
P\left\{X_{1}\right. & >Y\}=1-\int_{-\infty}^{\infty} F_{X_{1}}(y) \sum_{u=2}^{U}\left(\frac{d}{d y} F_{X_{u}}(y)\right) \prod_{\substack{k=2 \\
k \neq u}}^{U} F_{X_{k}}(y) d y \\
& =1-\sum_{u=2}^{U} \int_{-\infty}^{\infty} F_{X_{1}}(y)\left(\frac{d}{d y} F_{X_{u}}(y)\right) \prod_{\substack{k=2 \\
k \neq u}}^{U} F_{X_{k}}(y) d y \\
& =1-\sum_{u=2}^{U} \int_{-\infty}^{\infty}\left(\frac{d}{d y} F_{X_{u}}(y)\right) \prod_{\substack{k=1 \\
k \neq u}}^{U} F_{X_{k}}(y) d y
\end{aligned}
$$

Now, the integral in (15) will be evaluated by integration by parts $\left(\int_{a}^{b} u^{\prime} v=\left.[u v]\right|_{a} ^{b}-\int_{a}^{b} u v^{\prime}\right)$ :

$$
\begin{aligned}
& p_{u} \doteq \int_{-\infty}^{\infty}\left(\frac{d}{d y} F_{X_{u}}(y)\right) \prod_{\substack{k=1 \\
k \neq u}}^{U} F_{X_{k}}(y) d y \\
& \left.=F_{X_{u}}(y)\right)\left.\prod_{\substack{k=1 \\
k \neq u}}^{U} F_{X_{k}}(y)\right|_{-\infty} ^{\infty} \\
& -\int_{-\infty}^{\infty} F_{X_{u}}(y) \sum_{\substack{k=1 \\
k \neq u}}^{U}\left(\frac{d}{d y} F_{X_{k}}(y)\right) \prod_{\substack{n=1 \\
n \neq k \\
n \neq u}}^{U} F_{X_{n}}(y) d y \\
& =1-\sum_{\substack{k=1 \\
k \neq u}}^{U} \int_{-\infty}^{\infty} F_{X_{u}}(y)\left(\frac{d}{d y} F_{X_{k}}(y)\right) \prod_{\substack{n=1 \\
n \neq k \\
n \neq u}}^{U} F_{X_{n}}(y) d y \\
& =1-\sum_{\substack{k=1 \\
k \neq u}}^{U} \int_{-\infty}^{\infty}\left(\frac{d}{d y} F_{X_{k}}(y)\right) \prod_{\substack{n=1 \\
n \neq k}}^{U} F_{X_{n}}(y) d y \\
& =1-\sum_{\substack{k=1 \\
k \neq u}}^{U} p_{k}=p_{u}
\end{aligned}
$$

with (19) proving (5), and due to (20) we also have

$$
\sum_{k=1}^{U} p_{k}=1
$$

And, finally, (6) is obtained from (19) according to

$$
P\left\{X_{1}>Y\right\}=1-\sum_{u=2}^{U} p_{u}=p_{1} .
$$

The normalization property in (21) leads to the simplification in the calculation of $P\left\{X_{1}>Y\right\}$ in (22). 


\section{Outage Probability, Conditioned on $S_{1}=k$.}

The pdf of the "system-FOM" (as opposed to user-individual $F O M s$ ) that can be achieved system-wide, i.e. across all users, in the current transmit block (out of $N$ blocks we consider), can be found applying the maximum-order statistic theorems for i.n.i.d (independent, non-identically distributed) random variables. However, observing the FOMs that are assigned to a specific user in case of being scheduled reveals that these FOMs are not distributed according to the maximum-order statistic. Instead, it will be shown that the weighted pdfs of the FOMs assigned to the users will sum up to the maximumorder statistic of the system-FOM. The weights can be found by calculating the probabilities of the users being scheduled in a certain block $n$. With

$$
Z \doteq \max \left\{X_{1}, X_{2}, \ldots, X_{U}\right\},
$$

the cdf $F_{Z}(z)$ of the maximum of the system-FOMs (i.e. of the current FOMs of all users) can, for i.n.i.d. FOMs, be calculated as [9]:

$$
F_{Z}(z)=P\{Z \leq z\}=\prod_{u=1}^{U} P\left\{X_{u} \leq z\right\}=\prod_{u=1}^{U} F_{X_{u}}(z) .
$$

Calculating the pdf $f_{Z}(z)=\frac{d}{d z} F_{Z}(z)$ of (24) reveals the following structure:

$$
f_{Z}(z)=\sum_{u=1}^{U}\left[\left(\frac{d}{d z} F_{X_{u}}(z)\right) \prod_{\substack{k=1 \\ k \neq u}}^{U} F_{X_{k}}(z)\right]
$$

A comparison of (16) and (25) reveals structural similarity and suggests that the maximum order statistics of statistically independent (but not necessarily identically distributed) random variables is a linear combination of the statistics of the instances of the individual random variables which contribute to the maximum order statistics, weighted with the probability $p_{u}$ of each individual random variable to contribute the maximum. The probability $p_{1}$ was, however, computed above in (5) and (6) with the general form for any user $u$ given in (16). Hence, (25) is equivalently written as

$$
f_{Z}(z)=\sum_{u=1}^{U}\left[\tilde{f}_{X_{u}}(z) \cdot p_{u}\right] \text {. }
$$

with

$$
\tilde{f}_{X_{u}}(z)=\frac{\left(\frac{d}{d z} F_{X_{u}}(z)\right) \prod_{\substack{k=1 \\ k \neq u}}^{U} F_{X_{k}}(z)}{p_{u}}
$$

the pdf associated to the FOM of user $u$ in case this user is scheduled for transmission. The corresponding pdf of the FOM of the scheduled user 1 can therefore be written as

$$
\tilde{f}_{X_{1}}(z)=\frac{f_{X_{1}}(z) \cdot F_{X_{2}}(z) \cdot F_{X_{3}}(z) \ldots F_{X_{U}}(z)}{p_{1}}
$$

and be used to compute the outage probability

$$
P\left\{X_{1, \text { sum }}<x_{1, \text { sum }}^{\text {req }} \mid S_{1}=1\right\}=\int_{0}^{x_{1, \text { sum }}^{\text {req }}} \tilde{f}_{X_{1}}(x) d x
$$

for the case that user 1 is scheduled once $\left(S_{1}=1\right)$ within $N$ transmit blocks. For $S_{1}=2$ (i.e., user 1 being scheduled twice within $N$ blocks) the result reads

$$
P\left\{X_{1, \text { sum }}<x_{1, \text { sum }}^{\text {req }} \mid S_{1}=2\right\}=\int_{0}^{x_{1, \text { sum }}^{\text {req }}} \int_{0}^{\infty} \tilde{f}_{X_{1}}(\rho) \cdot \tilde{f}_{X_{1}}(x-\rho) d \rho d x
$$

as the pdf of a sum of two independent pdfs follows from a convolution of the pdfs. Consequently, for $S_{1}=k$, we obtain

$$
P\left\{X_{1, \text { sum }}<x_{1, \text { sum }}^{\text {req }} \mid S_{1}=k\right\}=\int_{0}^{x_{1, \text { sum }}^{\text {req }}} \underbrace{\left(\tilde{f}_{X_{1}} * \cdots * \tilde{f}_{X_{1}}\right)}_{k \text { times }}(x) d x
$$

where “*” denotes convolution.

\section{Results For SPECIFIC SCHEdulers AND CHANNELS}

In the following, the pdfs of the FOMs of opportunistic and proportional-fair scheduling are derived for the special case of user-individual Rayleigh-fading channels. The reason to pick Rayleigh-fading is that it allows for compact analytical expressions for the statistics of the FOMs, i.e., $f_{X_{1}}(z)$ and $F_{X_{u}}(z)$, that are used in (6) and (28): using the results from there, the outage probabilities are calculated by (29)-(31) and finally in (4) and (2). For the last two steps a numerical approach (incl. convolutions) is required anyway (as analytical solutions are intractable) so the whole scheme can as well be run with measured pdfs $f_{X_{1}}(z)$ and cdfs $F_{X_{u}}(z)$ that are used in the numerical implementations, e.g. of the integrations in (29)-(31). This is to say that, although the analytical results from Section III are demonstrated for simple Rayleigh fading, they could equally be applied for any measured channel statistics, so the method as such is not restricted to any specific simple channel model.

\section{A. Opportunistic Scheduling}

If a channel-coefficient $h$ is Rayleigh-distributed with scaleparameter $\lambda$, the pdf can be expressed as:

$$
f_{H}(h)=\frac{h}{\lambda^{2}} \mathrm{e}^{-\frac{h^{2}}{2 \lambda^{2}}}, \quad h>0 .
$$

According to elementary probability calculus (e.g. [10, p. 130]) the magnitude-square $|H|^{2}$ has the exponential distribution

$$
f_{|H|^{2}}\left(|h|^{2}\right)=\frac{1}{2 \lambda^{2}} \mathrm{e}^{-\frac{|h|^{2}}{2 \lambda^{2}}} .
$$

If the scheduler applies a classic opportunistic scheme (i.e. decides in favor of the user with the highest achievable rate), it applies a $\max$-operation on the rates for all users. Therefore, the pdf of the rate $r$ needs to be determined. With

$$
r=g\left(|h|^{2}\right)=\log _{2}\left(1+\frac{P}{2 \sigma^{2}}|h|^{2}\right)
$$

(Gaussian channel capacity for a fixed channel coefficient $h$ ) and the derivative

$$
g^{\prime}\left(|h|^{2}\right)=\frac{1}{\log (2)} \cdot \frac{\frac{P}{2 \sigma^{2}}}{|h|^{2} \frac{P}{2 \sigma^{2}}+1},
$$


the standard pdf-transformation (e.g. [10])

$$
f_{R}(r)=f_{|h|^{2}}\left(|h|^{2}\right) /\left.g^{\prime}\left(|h|^{2}\right)\right|_{|h|^{2}=g^{-1}(r)}
$$

(note that $g\left(|h|^{2}\right)$ is monotonic) results in

$$
f_{R}\left(|h|^{2}\right)=\log (2) \cdot \frac{|h|^{2} \frac{P}{2 \sigma^{2}}+1}{\frac{P}{2 \sigma^{2}}} \cdot \frac{1}{2 \lambda^{2}} \cdot \mathrm{e}^{\frac{-|h|^{2}}{2 \lambda^{2}}},
$$

and substituting $|h|^{2}=\frac{2 \sigma^{2}}{P}\left(2^{r}-1\right)$ leads to the rate-pdf

$$
f_{R}(r)=\log (2) \beta \cdot 2^{r} \cdot \mathrm{e}^{-\beta\left(2^{r}-1\right)} .
$$

with

$$
\beta=\frac{\sigma^{2}}{\lambda^{2} P}
$$

The cdf follows by direct integration of (38); it reads

$$
F_{R}(r)=1-e^{-\beta\left(2^{r}-1\right)} .
$$

The pdf (38) and the cdf (40) are to be used in place of $f_{X_{1}}(z)$ and $F_{X_{u}}(z)$ in (6) and (28) to compute $p_{1}$ and $\tilde{f}_{X_{1}}(z)$ respectively. The pdf and the cdf only depend on the parameter $\beta$ in (39), which contains the transmit power $P$, the parameter $\lambda$ of the fading process and noise variance $\sigma^{2}$ at the receiver. All those parameters can (and will in practice) be different for every user.

Using a Matlab implementation, Figure 1 illustrates the outage probability for a system with 5 users, a window-length of 10 and 20 blocks and transmit SNRs $\left(=10 \log _{10} \frac{P}{2 \sigma^{2}}\right)$ of 0,10 and $20 \mathrm{~dB}$. The fading statistics of all users are assumed to be Rayleigh-distributed with scale-parameters $\lambda=[2,2,3,4,5]$ for the 5 users, respectively. The $\mathrm{x}$-axis is the sum-rate across all blocks in a window of size $N(=10,20)$ that is achieved by user 1 . This sum rate adds the rates in "bits per channeluse" according to (34) from the individual blocks (all of which are assumed to be infinitely long by the common block fading model) within a window of size $N$ blocks: this approach has been chosen to separate the curves in the plots (and, hence improve readability). In practice one could normalise the sumrate axis by the window length $N$ to obtain a normalized average rate in bits per channel use. The probability of outage was calculated for user 1 by the method described in Section III for transmit SNRs of $(0,10,20 \mathrm{~dB}$ from left to right in the curves). The solid curves stem from a Monte-Carlo simulation approach, and the individually marked points were calculated according to the analytical approach stated above. As to be expected, the figure shows that higher SNR and larger windowsize $N$ always cause a lower outage probability. The figure also demonstrates that the analytical approach (with numerical computations of integrals and convolutions) is rather accurate as the marked points computed are sitting almost exactly on the curves found by extensive Monte-Carlo simulations.

\section{B. Proportional Fair Scheduling}

Opportunistic scheduling and proportional fair scheduling are similar schemes in the sense that both select the user with the highest FOM. For opportunistic scheduling, the FOM is the achievable rate in the current block; for proportional-fair

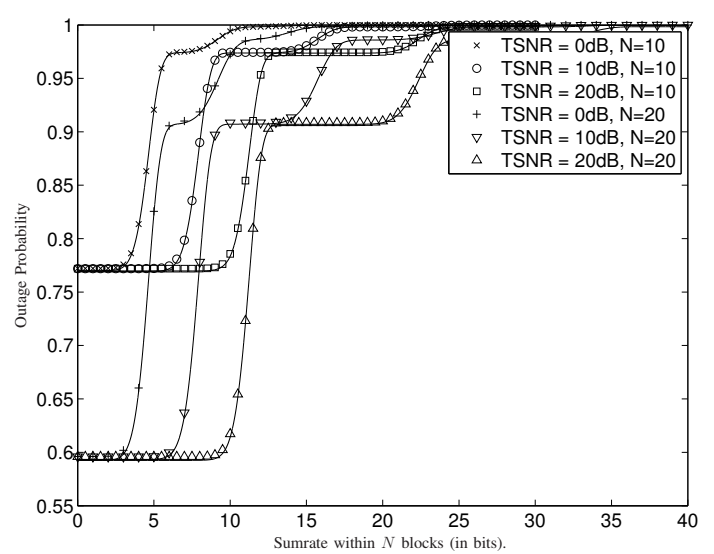

Fig. 1. Outage probability of user 1 for 5-user opportunistic scheduling in a Rayleigh-fading environment with transmit-SNRs OdB, $10 \mathrm{~dB}$, and $20 \mathrm{~dB}$ and window-lengths of 10 and 20 blocks. Numerical results based on $10^{6}$ simulated windows of length $N$ (solid lines) are compared with outage probabilities computed analytically (individually marked points).

scheduling, the FOM is the rate that can be achieved in the current block, divided by the "recently achieved" rate: a user is scheduled, if their ratio is larger than for any other user. We will model this scheme by using a long-term average for the normalization of the current rate. Since there is currently no universally-valid closed-form expression for the long-term average achieved rate known for proportional-fair scheduling, we use the iterative solution suggested in [6] and refined in [11]:

$$
E\left\{T_{1}\right\}=\int_{0}^{\infty} r \cdot f_{R_{1}}(r) \prod_{u=2}^{U} F_{R_{u}}\left(r \cdot \frac{E\left\{T_{u}\right\}}{E\left\{T_{1}\right\}}\right) d r .
$$

This expression has been shown by [12] to converge to the measured mean throughput of $u_{1}$, if the instantaneous rate follows a continuous distribution.

Therefore, $u_{1}$ 's figure of merit for PF-scheduling is

$$
\bar{R}_{1}=\frac{R_{1}}{T_{1}}
$$

Then, the pdf of user 1's FOM can be written as (compare (38)):

$$
f_{\bar{R}_{1}}\left(\bar{r}_{1}\right)=T_{1} \cdot \log (2) \beta_{1} \cdot 2^{T_{1} \bar{r}_{1}} \cdot \mathrm{e}^{-\beta_{1}\left(2^{T_{1} \bar{r}_{1}}-1\right)}
$$

Again, this pdf is to be used in (29), in place of $f_{X_{1}}(z)$. The following Figure 2 illustrates the precision of the approach. The simulation parameters chosen are the same as in the opportunistic scheduling case. Please note that a proportionalfair scheduler updates the average-achieved rates $T_{u}$ of the served users by applying an exponentially-weighted movingaverage (EWMA) filter. Since in this paper, the expected value for the average-achieved rates $T_{u}$ was used, the EWMAfilter in the simulations was assigned a long memory, i.e., a weighting-factor of $\alpha=10^{5}$.

The presented results for opportunistic and proportional-fair scheduling demonstrate the accuracy of the approach introduced in this paper. It should be noted that, although a 


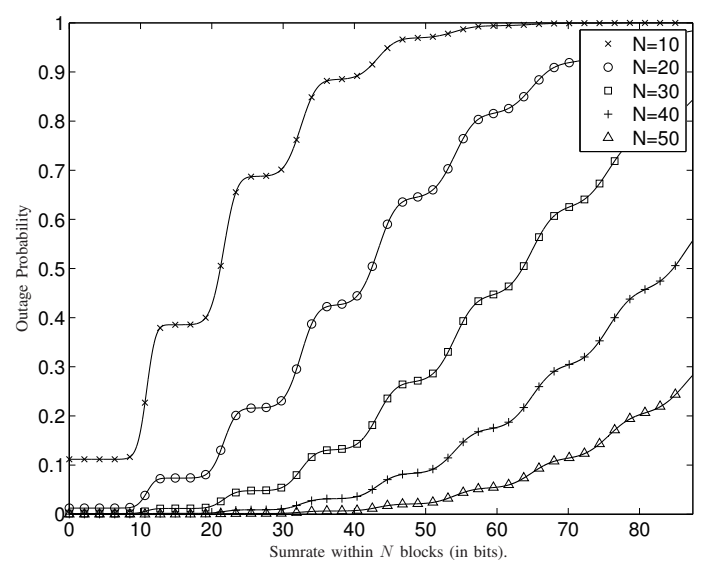

Fig. 2. Outage probability of user 1 for 5 -user proportional-fair scheduling in a Rayleigh-fading environment with a transmit-SNR of $10 \mathrm{~dB}$ and windowlengths of $10,20,30,40$, and 50 blocks. Numerical results based on $10^{7}$ simulated blocks and an EWMA weighting-factor of $10^{5}$ (solid lines) are compared to outage probabilities computed analytically (individually marked points).

Rayleigh-fading channel was assumed for all users (albeit with different parameters for each user), the presented approach is by no means limited to a Rayleigh-fading environment. This was only chosen for mathematical tractability and ease of analysis. The presented principles can be applied to any scheduler which assigns resources based on a max-operation. In that, the approach is highly general, but at the same time, as demonstrated, also practically useful. Especially in a world of multimedia transmissions, the possibility of evaluating one of the most highly regarded wireless schedulers, the proportional fair, for outage probability without any principle restrictions on system parameters is in our opinion highly valuable. Based on the insights of this paper, the question if a numerical simulation is long enough for the results to be sufficiently accurate is no longer necessary. Due to the considerable processing time of the algorithm, we do not propose to generate a "smooth" outage-plot, but to use it as a benchmark for assessing the numerical accuracy of simulations.

\section{CONCLUSIONS}

This paper introduces an novel analytical approach to calculate the outage probability - the probability that a certain raterequest can not be met - of max-based scheduling under delay constraints.
The problem is solved in a most general fashion, neither restricting the number of users to be served by the basestation, their channel statistics nor their delay constraints. It is shown that the presented approach is also applicable to min-based schedulers; the demonstration, however, is carried out only for the max-based opportunistic and proportional fair schemes. To prove the correctness of the approach, results of analytical calculations and extensive numerical (Monte-Carlo) simulations are found to match accurately. Furthermore, in the course of this work, a new and useful interpretation of the mathematical structure of the maximum order statistic for i.n.i.d. random variables (that is also valid for the special case of i.i.d random variables) has been found and exploited to obtain the results.

\section{REFERENCES}

[1] R. Knopp and P.A. Humblet, "Information capacity and power control in single-cell multiuser communications," in Proceedings IEEE International Conference on Communications (ICC), Seattle, WA, USA, June 1995, pp. 331-335.

[2] P. Viswanath, D.N.C. Tse, and R. Laroia, "Opportunistic beamforming using dumb antennas," IEEE Transactions on Information Theory, vol. 48, no. 6, pp. 1277-1294, June 2002.

[3] S.V. Hanly and D.N.C. Tse, "Multi-access fading channels: Delaylimited capacities," in Proceedings IEEE International Symposium on Information Theory, Aug. 1998, p. 397.

[4] R. Knopp G. Caire, R.R. Müller, "Hard fairness versus proportional fairness in wireless communications: The single cell case," IEEE Transactions on Information Theory, vol. 53, no. 4, pp. 1366-1385, Apr. 2007.

[5] J.-G. Choi and S. Bahk, "Cell-throughput analysis of the proportional fair scheduler in the single-cell environment," IEEE Transactions on Vehicular Technology, vol. 56, no. 2, pp. 766-778, Mar. 2007.

[6] J.M. Holtzman, "Asymptotic analysis of proportional fair algorithm," in Proceedings IEEE International Symposium on Personal, Indoor and Mobile Radio Communications, Sept./Oct. 2001, vol. 2, pp. F-33 - F-37 vol. 2.

[7] E. Liu, Q. Zhang, and K.K. Leung, "Asymptotic analysis of proportionally fair scheduling in Rayleigh fading," IEEE Transactions on Wireless Communications, vol. 10, no. 6, pp. 1764-1775, June 2011.

[8] E. Liu and K.K. Leung, "Proportional fair scheduling: Analytical insight under Rayleigh fading environment," in Proceedings IEEE Wireless Communications and Networking Conference (WCNC), Apr. 2008, pp. 1883-1888.

[9] H.A. David and H.N. Nagaraja, Order Statistics, 3rd Edition, Wiley \& Sons, 3rd edition, 2003.

[10] A. Papoulis and S.U. Pillai, Probability, Random Variables and Stochastic Processes, vol. Fourth Edition, McGraw-Hill, 2002.

[11] G. Horvath and C. Vulkan, "Throughput analysis of the proportional fair scheduler in HSDPA," in Wireless Conference, 2008. EW 2008. 14th European, June 2008.

[12] H.J. Kushner and P.A. Whiting, "Convergence of proportional-fair sharing algorithms under general conditions," Wireless Communications, IEEE Transactions on, vol. 3, no. 4, pp. 1250 - 1259, July 2004. 